\title{
Recurrent Desmoid-Type Fibromatosis
}

National Cancer Institute

\section{Source}

National Cancer Institute. Recurrent Desmoid-Type Fibromatosis. NCI Thesaurus. Code C155875.

Desmoid-type fibromatosis that has recurred after a period of remission. 